\title{
Advanced BPANN Based Modified Boundary Discriminative Noise Removal Technique for Salt and Pepper Noise Removal: A Survey
}

\author{
Apoorva Agarwal, Mr. Pankaj Kumar
}

\begin{abstract}
Noise removal is one of the greatest challenges among the researchers, noise removal algorithms vary with the application areas and the type of images and noises. Noise can degrade the image at the time of capturing or transmission of the image. Before applying image processing tools to an image, noise removal from the images is done at highest priority Therefore, relatively fewer works have been published in this area. In this paper the novel approach has been presented for removal of salt and pepper noise from the high density salt $\&$ pepper noisy images, using Iterative Modified Decision based Unsymmetric Trimmed Median Filter. The existing MDBUTMF is unable to restore the original image from the noisy one if noise density is more than $70 \%$.
\end{abstract}

\section{INTRODUCTION}

Image restoration is the process of restoring the original image from the observed image that can be degraded or blurred by some external sources. In image restoration, the main emphasis is to obtaining a deblurred image from a corresponding blurred one. There are numbers of factors that are responsible for blurring in the image like atmospheric turbulence, bad focus and motion between camera and original image. Generally when intensity of an image point is spread over several pixels, it goes blurred. This blurring is defined by a well-known operator called Point Spread Function (PSF). Generally blur in the image is not uniform over an entire image. The amount of blur is different in different directions. The maximum amount of blur in the image is at the center of the image. So it is necessary to deblur the center part of an image compared to corner segments. De-noising is one of the basic problems in image processing. For example, interpolation or resizing is to estimate plausible pixel values located between known ones while de-noising or de-blurring is to estimate clean pixel values from corrupted ones.

Filling missing parts of an image in order to obtain a visually plausible outcome is the problem addressed in three distinct but related fields of study [1]. Image de-noising is an important image processing task, both as a process itself, and as a component in other processes. Very many ways to de-noise an image or a set of data exists. The main property of a good image de-noising model is that it will remove noise while preserving edges. Traditionally, linear models have been used. One common approach is to use a Gaussian filter, or equivalently solving the heat-equation with the noisy image as input-data, i.e. a linear, 2nd order PD Emodel [2]. For some purposes this kind of de-noising is adequate. One

Apoorva Agarwal, M.Tech Scholar, Department of Computer Science \& Engineering, Shri Ramswaroop Memorial College of Engineering and Management, Lucknow, India.

Mr. Pankaj Kumar, Associate Professor, Department of Computer Science \& Engineering, Shri Ramswaroop Memorial College of Engineering and Management, Lucknow, India. big advantage of linear noise removal models is the speed. But a drawback of the linear models is that they are not able to preserve edges in a good manner: edges, which are recognized as discontinuities in the image, are smeared out. Nonlinear models on the other hand can handle edges in a much better way than linear models can. One popular model for nonlinear image de-noising is the Total Variation (TV) filter, introduced by Rudin, Osher and Fatemi. This filter is very good at preserving edges, but smoothly varying regions in the input image are transformed into piecewise constant regions in the output image [3].

The noisy pixel is replaced by the median/mean/mid-point value of the window or by its neighborhood values. For high density salt and pepper noise it might so happen that the replaced pixel (median/mean) might be a noisy pixel which does not help in suppression of noise. The Modified Decision Based Unsymmetric Trimmed Median Filter replaces the noisy pixel by the trimmed median value (excluding the either 0 or 255 the noise pixel is replaced by the mean value of all the elements present in the current window [4].

\section{VARIOUS SORCES OF NOISE IN IMAGES}

Noise is introduced in the image at the time of image acquisition or transmission. Different factors may be responsible for introduction of noise in the image. The number of pixels corrupted in the image will decide the quantification of the noise. The principal sources of noise in the digital image are:

a) The imaging sensor may be affected by environmental conditions during image acquisition.

b) Insufficient Light levels and sensor temperature may introduce the noise in the image.

c) Interference in the transmission channel may also corrupt the image.

d) If dust particles are present on the scanner screen, they can also introduce noise in the image.

\section{VARIOUS NOISE TYPES:-}

Noise is the undesirable effects produced in the image. During image acquisition or transmission, several factors are responsible for introducing noise in the image. Depending on the type of disturbance, the noise can affect the image to different extent. Generally our focus is to remove certain kind of noise. So we identify certain kind of noise and apply different algorithms to remove the noise. Image noise can be classified as Impulse noise (Salt-and-pepper noise), Amplifier noise (Gaussian noise), Shot noise, Quantization 


\section{Advanced BPANN Based Modified Boundary Discriminative Noise Removal Technique For Salt and Pepper Noise Removal: A Survey}

noise (uniform noise), Film grain, on-isotropic noise, Multiplicative noise (Speckle noise) and Periodic noise.

Impulse Noise (Salt and Pepper Noise): The term impulse noise is also used for this type of noise [5]. Other terms are spike noise, random noise or independent noise. Black and white dots appear in the image [6] as a result of this noise and hence salt and pepper noise. This noise arises in the image because of sharp and sudden changes of image signal. Dust particles in the image acquisition source or over heated faulty components can cause this type of noise. Image is corrupted to a small extent due to noise. Fig. 1 (a) and 1 (b) shows respectively the original Lena image and the salt and pepper noise affected image with variance 0.05 .

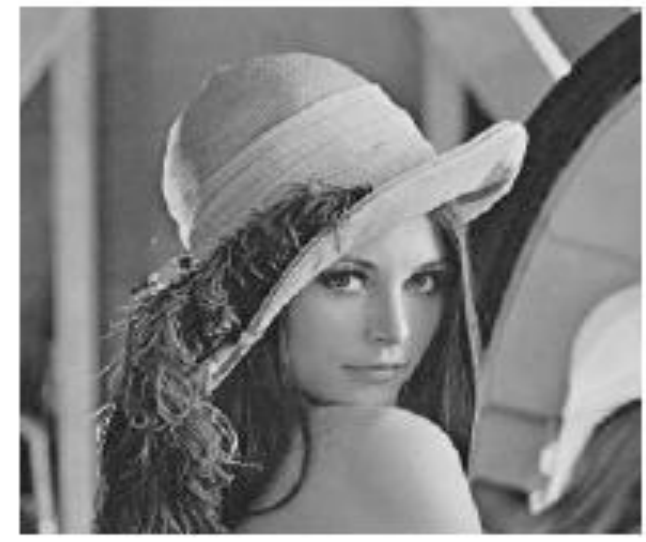

Figure 1(a): Original Image

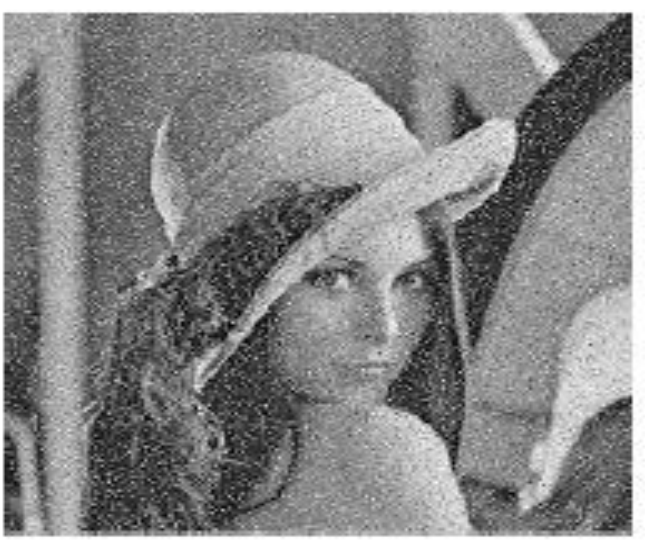

Figure 1(b): Sault and pepper noise

The image may not be fully corrupted due to this noise, but some pixel values will change. The corresponding value for black pixel will be extremely low i.e., 0 and the corresponding value for white pixel will be extremely high i.e., 1. This noise can be eliminated by using dark frame subtraction and interpolating around dark/bright pixels.

Gaussian Noise (Amplifier Noise): The term normal noise model is the synonym of Gaussian noise. This noise model is additive in nature [7] and follow Gaussian distribution. Meaning that each pixel in the noisy image is the sum of the true pixel value and a random, Gaussian distributed noise value. The noise is independent of intensity of pixel value at each point. An example of Gaussian noise affected image with mean 0 and variance 0.5 is shown in Fig. 2.

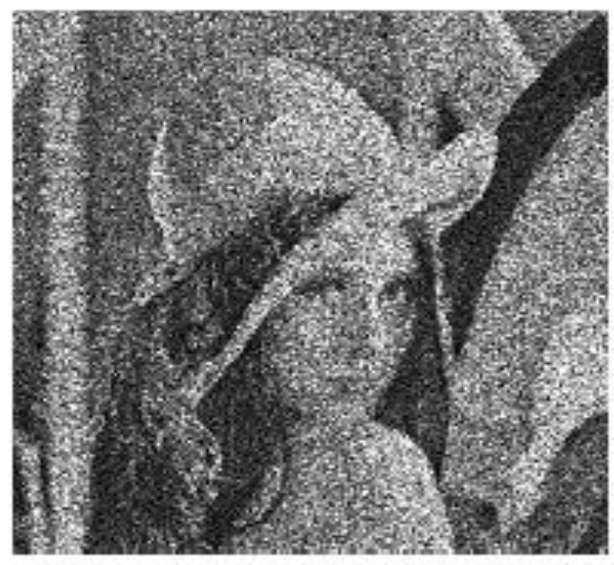

Figure 2: Gaussian Noise

$($ mean $=0$, variance $=0.05)$

Speckle Noise: This noise is originated because of coherent processing of back scattered signals from multiple distributed points. In conventional radar system this type of noise is noticed when the returned signal from the object having size less than or equal to a single image processing unit, shows sudden fluctuations. An example of speckle noise on an image with variance 0.5 is shown in Fig. 3 .

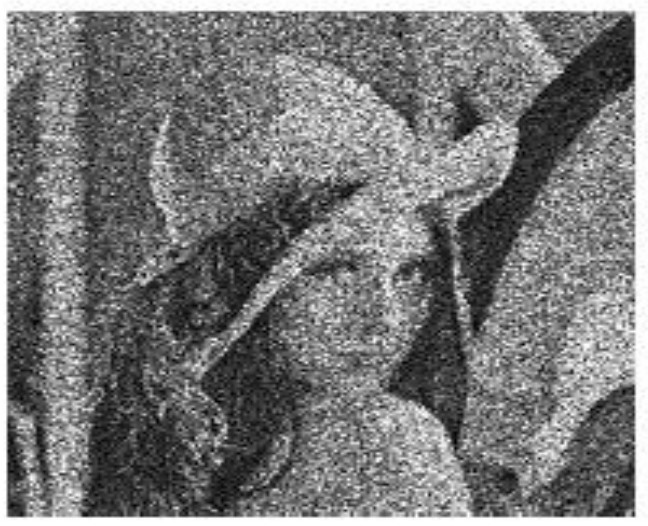

Figure 3: Speckle noise

\section{IMAGE DE-NOISING}

Image de-noising is very important task in image processing for the analysis of images. Ample image de-noising algorithms are available, but the best one should remove the noise completely from the image, while preserving the details. De-noising methods can be linear as well as non-linear. Where linear methods are fast enough, but they do not preserve the details of the images, whereas the non- linear methods preserve the details of the images. Broadly speaking,

De-noising filters can be categorized in the following categories:

- Adaptive Filter

- Order Statistics Filter

- Mean Filter

- Averaging

- Median Filter

Adaptive Filter:- These filters change their behavior on the basis of statistical characteristics of the image region, 
encompassed by the filter region.BM3D is an adaptive filter. It is a nonlocal image modeling technique based on adaptive, high order group-wise models. This de-noising algorithm can be divided in three steps [7-8]:

1. Analysis. Firstly similar image blocks are collected in groups. Blocks in each group are stacked together to form 3-D data arrays, which are de-correlated using an invertible 3D transform.

2. Processing. The obtained 3-D group spectra are filtered by hard thresholding.

3. Synthesis. The filtered spectra are inverted, providing estimates for each block in the group. These block-wise estimates are returned to their original positions and the final image reconstruction is calculated as a weighted average of all the obtained block-wise estimates.

Order Statistics Filter:- Order-Statistics filters are non-linear filters whose response depends on the ordering of pixels encompassed by the filter area. When the center value of the pixel in the image area is replaced by 100th percentile, the filter is called max-filter. On the other hand, if the same pixel value is replaced by 0th percentile, the filter is termed as minimum filter. In this filtering technique, the pixel is replaced with the median of the neighboring pixels. A window is chosen, which vary for the $1 \mathrm{D}$ signal and 2D signals, and the window slides over each pixel value. Some issues with median filter includes that the majority of the computational effort and time is spent on calculating the median of each window.

\section{Mean Filter:-}

Mean filter is an averaging linear filter [6]. Here the filter computes the average value of the corrupted image in a predecided area. Then the center pixel intensity value is replaced by that average value. This process is repeated for all pixel values in the image.

\section{Averaging Filter:-}

The averaging filter is used to restoring gray scale and color images highly corrupted by salt and pepper noise and overcoming the drawback of mean filter. As in mean filter here also first the corrupted pixel is detected and then one of the below case is applied to that pixel:

Case 1: If the selected window contains noisy pixel (255 or 0 ) and all the neighboring pixel values are also noisy pixels, then their median value will also be noisy. Hence to avoid this, the mean is calculated of the pixels in the selected window and the noisy pixel is replaced by that value.

Case 2: If the selected window contains noisy pixel (255 or 0 ) and some of the neighboring pixel values are noisy, then their median value will also be noisy. Hence to remove noise from the image, 1-D array of the selected image region is obtained so that the $0 / 255$ values will be eliminated and after this the median of remaining values is calculated and the noisy pixel value is replaced by this value.

Case 3: If there is no noisy pixel in the selected window, then no changes are done and the pixel value is left unchanged. This algorithm shows better results than the other filters but the drawback is that it leads to blurring of the image at higher noise densities.

\section{Median Filter:-}

Median filter is a best order static, non- linear filter, whose response is based on the ranking of pixel values contained in the filter region. Median filter is quite popular for reducing certain types of noise. Here the center value of the pixel is replaced by the median of the pixel values under the filter region [9] [10]. The median filter is a non-linear filtering technique which is used to remove noise. In this filtering technique, the pixel is replaced with the median of the neighboring pixels. A window is chosen, which vary for the 1D signal and 2D signals, and the window slides over each pixel value. Some issues with median filter includes that the majority of the computational effort and time is spent on calculating the median of each window. As the filter must process every entry in the signal therefore for large signals, the efficiency of median calculation is a critical in determining how fast the algorithm can run. Also median filter is only effective at low noise densities and fails at higher noise densities

\section{MODIFIED MEDIAN FILTER}

The proposed Modified Decision Based Unsymmetric Trimmed Median Filter (MDBUTMF) [6] algorithm processes the corrupted images by first detecting the impulse noise. The processing pixel is checked whether it is noisy or noisy free. That is, if the processing pixel lies between maximum and minimum gray level values then it is noise free pixel, it is left unchanged. If the processing pixel takes the maximum or minimum gray level then it is noisy pixel which is processed by MDBUTMF. The steps of the MDBUTMF are elucidated as follows.

Step 1: Insert 0's to the first row, First column and last row, last column of the image.

Step 2: Select a window of size $3 \times 3$, and consider the processing pixel is pij in the window.

Step 3: Processing the corrupted image:

If the processing pixel value lies between $0<$ $\mathrm{P}_{\mathrm{ij}}<255$, then it is an uncorrupted pixel and its value is left unchanged.

Step 4: if $\mathrm{P}_{\mathrm{ij}}=0$ or 255 , then $\mathrm{P}_{\mathrm{ij}}$ is a corrupted pixel. The possible cases of processing the pixel:

Case (i): If the selected window contains all 0's and 255 's, then $\mathrm{P}_{\mathrm{ij}}$ is replaced with mean of the element of the window.

Case (ii): If all the elements in the selected window does not have 0 's and 225 's,

Eliminate 0's and 255's, sort in the ascending order and find the median value of the remaining elements. Replace pij with the median value.

Step 5: Repeat steps 2 to 4 until all the pixel in the entire image is processed.

Step 6: Repeat steps 2 to 5.

Step 7: Remove additionally inserted rows \& columns of 0's in step 1 .

\section{IMAGE RESTORATION}

In super resolution process image reconstruction or we can say restoration is final and very crucial step. It removes blurring effect and also the noise present in the image. The general model or principle can be represented as

$$
\mathrm{g}(\mathrm{x}, \mathrm{y})=\mathrm{h}(\mathrm{x}, \mathrm{y}) * \mathrm{f}(\mathrm{x}, \mathrm{y})+\mathrm{n}(\mathrm{x}, \mathrm{y})
$$

Where $g(x, y)$ is the low resolution image, $h(x, y)$ is PSF (point spread function), $f(x, y)$ is the high resolution ideal image, $n(x, y)$ is noise present in the image.

Fourier transforms (FT) of equation 1 is given by

$$
\mathrm{G}(\mathrm{u}, \mathrm{v})=\mathrm{H}(\mathrm{u}, \mathrm{v}) \mathrm{F}(\mathrm{u}, \mathrm{v})+\mathrm{N}(\mathrm{u}, \mathrm{v})
$$




\section{Advanced BPANN Based Modified Boundary Discriminative Noise Removal Technique For Salt and Pepper Noise Removal: A Survey}

Super resolution $(\mathrm{SR})$ restoration is to perform analytic continuation to $F(u, v)$ to extend its support domain by applying prior information and posterior processing technologies.

Now get new point spread function (PSF)H' (u, v). H(u, v) also has the extended support domain; therefore the resolution of the image is enhanced. There is usually very small prior information in images and the point spread function of the image is tedious to get, so blind deconvolution is most probably used to reconstruct the image.

Typical blind deconvolutions are of two types

1) The recognition of point spread function is separated with the restoration of image. Point spread function (PSF) is obtained first, after that traditional restoration methods are used to compute the estimate of original image.

2) The recognition of PSF (point spread function) and restoration of image are performed at the same time, so this kind of method is very complex.

Beside this there are some other approach which are widely used in blind deconvolution are Auto Regressive Moving Average and Priori Blur Identification method.

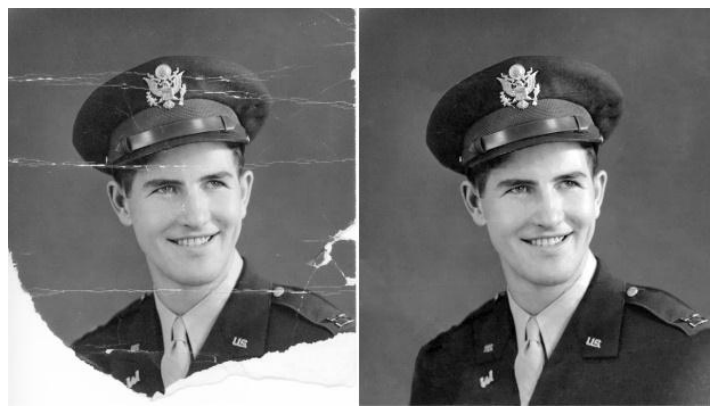

Fig 3.8 Image after restoration

\section{CONCLUSION}

In this paper, various denoising techniques are discussed. The denoising methods are broadly classified for ease of understanding. In each category a brief idea of the different existing denoising methods are presented.

A new algorithm Advanced modified decision based unsymmetrical trimmed median filter (AMDBUTMF) is proposed and developed for different de noising images of different format. Whereas, averaging and minimum filters performed worst. Median filter is the best choice of removing the Salt and pepper noise. In further work of my dissertation is modified median filter and improved PSNR (peak signal noise ratio) and reduced mean square error (MSE) for gray an color image.

The performance of this method is tested for different noise densities with gray scale images. Particularly at high noise densities the proposed method is better in removing the effect of noise. This method is also applicable for another type of noises like speckle, Gaussian, random etc.

\section{REFERENCES}

[1] Chauhan, Arjun Singh, and Vineet Sahula. "High density impulsive Noise removal using decision based iterated conditional modes" in Signal Processing, Computing and
Control (ISPCC), 2015 International Conference on, pp. 2429. IEEE, 2015.

[2] Dash, Arabinda, and Sujaya Kumar Sathua. "High Density Noise Removal by Using Cascading Algorithms" in Advanced Computing \& Communication Technologies (ACCT), 2015 Fifth International Conference on, pp. 96101. IEEE, 2015.

[3] Utaminingrum, Fitri, Keiichi Uchimura, and Gou Koutaki. "High density impulse noise removal based on linear meanmedian filter" in Frontiers of Computer Vision,(FCV), 2013 19th Korea-Japan Joint Workshop on, pp. 11-17. IEEE, 2013.

[4] Ashutosh Pattnaik, Sharad Agarwal and Subhasis Chand. "A New and Efficient Method for Removal of High Density Salt and Pepper Noise Through Cascade Decision based Filtering Algorithm" in 2nd International Conference on Communication, Computing \& Security, Volume 6, Pages 108-117. ICCCS-2012.

[5] Raza, Md Tabish, and Suraj Sawant. "High density salt and pepper noise removal through decision based partial trimmed global mean filter" in Engineering (NUiCONE), 2012 Nirma University International Conference on, pp. 1- 5. IEEE, 2012.

[6] Madhu S. Nair and G. Raju. "A new fuzzy-based decision algorithm for high-density impulse noise removal" in Signal, Image and Video Processing, November 2012, Volume 6, Issue 4, pp 579-595.

[7] Esakkirajan, S., T. Veerakumar, Adabala N. Subramanyam, and Prem $\mathrm{CH}$ Chand. "Removal of high density salt and pepper noise through modified decision based unsymmetric trimmed median filter" in Signal Processing Letters, IEEE 18, no. 5 (2011): 287-290.

[8] Aiswarya, K., V. Jayaraj, and D. Ebenezer. "A new and efficient algorithm for the removal of high density salt and pepper noise in images and videos" in Computer Modeling and Simulation, 2010. ICCMS'10. Second International Conference on, vol. 4, pp. 409-413. IEEE, 2010.

[9] V. Jayaraj and D. Ebenezer. "A New Switching-Based Median Filtering Scheme and Algorithm for Removal of High-Density Salt and Pepper Noise in Images" in EURASIP Journal on Advances in Signal Processing, 2010.

[10] D. Ebenezer, V. Jayaraj, and K. Aiswarya. "High Density Salt and Pepper Noise Removal in Images using Improved Adaptive Statistics Estimation Filter" in IJCSNS International Journal of Computer Science and Network Security, VOL.9 No.11, November 2009.

[11] V.R.Vijaykumar, P.T.Vanathi, P.Kanagasabapathy, and D.Ebenezer. "High Density Impulse Noise Removal Using Robust Estimation Based Filter" in IAENG Internal Journal of Computer Science, 35:3, in 2008.

[12] Srinivasan, K. S., and David Ebenezer. "A new fast and efficient decision-based algorithm for removal of highdensity 
impulse noises" in Signal Processing Letters, IEEE 14, no. 3 (2007): 189-192.

[13] S. Schulte, M. Nachtegael, V. De Witte, D. Van der Weken, and E. E. Kerre. A fuzzy impulse noise detection and reduction method. IEEE Trans. on Image Processing, 15(5):1153-1162, May 2006.

Apoorva Agarwal, M.Tech Scholar, Department of Computer Science \& Engineering, Shri Ramswaroop Memorial College of Engineering and Management, Lucknow, India.

Mr. Pankaj Kumar, Associate Professor, Department of Computer Science \& Engineering, Shri Ramswaroop Memorial College of Engineering and Management, Lucknow, India. 